\title{
Reconstruction of antinucleus-annihilation events in the GAPS experiment
}

\author{
Alessio Tiberio $^{a, *}$ on behalf of the GAPS Collaboration \\ (a complete list of authors can be found at the end of the proceedings) \\ ${ }^{a}$ INFN, section of Florence \\ Via Bruno Rossi 1, Sesto Fiorentino (FI), Italy \\ E-mail: alessio.tiberio@fi.infn.it
}

The General Antiparticle Spectrometer (GAPS) experiment is designed to detect low-energy $(<0.25 \mathrm{GeV} / n)$ cosmic-ray antinuclei as indirect signatures of dark matter. Several beyondthe-standard-model scenarios predict a large antideuteron flux due to dark matter decay or annihilation compared to the astrophysical background. The GAPS experiment will perform such measurements using long-duration balloon flights over Antarctica, beginning in the 2022/23 austral summer. The experimental apparatus consists of ten planes of $\mathrm{Si}(\mathrm{Li})$ detectors surrounded by a time-of-flight system made of plastic scintillators. The detection of the primary antinucleus relies on the reconstruction of the annihilation products: the low-energy antinucleus is captured by an atom of the detector material, forming an exotic atom that de-excites by emitting characteristics X-rays. Finally, the antinucleus undergoes nuclear annihilation, producing a "star" of pions and protons emitted from the annihilation vertex. Several algorithms were developed to determine the annihilation vertex position and to reconstruct the topology of the primary and secondary particles. An overview of the event reconstruction techniques and their performances, based on detailed Monte Carlo simulation studies, will be presented in this contribution.

$37^{\text {th }}$ International Cosmic Ray Conference (ICRC 2021)

July 12th - 23rd, 2021

Online - Berlin, Germany

\footnotetext{
${ }^{*}$ Presenter
} 


\section{Introduction}

Since the first detection of antiprotons in the 1970s, cosmic-ray antinuclei have been used as a tool to search for dark matter signatures or other new astrophysical phenomena. Antiproton measurements appear to be consistent with secondary production, but a contribution from dark matter annihilation or decay cannot be completely excluded $[1,2]$. On the other hand, heavier antinuclei as antideuteron and antihelium can provide a much clearer evidence for dark matter signature: in the energy region below few $\mathrm{GeV} / n$, several beyond-the-Standard Model theories predict a contribution from dark matter annihilation or decay several orders of magnitude greater than the astrophysical background [3,4]. Currently only upper limits have been provided for antideuteron spectrum in cosmic rays (as in [5]).

The General AntiParticle Spectrometer (GAPS) $[6,7]$ is the first experiment designed for the observation of low-energy antinuclei, covering the energy region below $0.25 \mathrm{GeV} / n$. As opposed as other experiments, which are based on a magnetic spectrometer, GAPS will use a new identification technique based on the formation of an exotic atom and the observation of its decay and annihilation products. The GAPS experiment will perform such measurements using long-duration balloon flights over Antarctica. At least three flights are planned and the first is expected to be performed in the 2022/23 austral summer. During its lifetime, GAPS will improve the sensitivity for antideuteron and antihelium nuclei by at least two orders of magnitude [8, 9]. Moreover, GAPS will collect the largest sample of antiprotons to date and it will extend the energy coverage in the unexplored region below $100 \mathrm{MeV}$.

The algorithms developed for the reconstruction of the topology of the antinucleus annihiliation inside the detector are described in this paper. In Section 2 a brief description of the experimental apparatus is given. The reconstruction algorithms are then explained in Section 3 and the obtained reconstruction performances are summarized in Section 4.

\section{The GAPS experiment}

The GAPS experimental apparatus consists of a time-of-flight (ToF) system surrounding several tracker planes. The ToF is arranged in an outer and an inner ToF systems made of $\sim 160$ plastic scintillator paddles. The outer ToF if made of and horizontal plane above the rest of the detector (named "umbrella") and of four lateral vertical walls (named "cortina"). The inner ToF is a cube that surround the tracker system on top, bottom and lateral sides. All scintillators are $6.35 \mathrm{~mm}$ thick and $16 \mathrm{~cm}$ wide, with a variable lenght between 1.51 and $1.8 \mathrm{~m}$. Each paddle system provides the measurement of energy deposit, time and longitudinal position along its largest dimension [10]. The tracker systems is made of $1440 \mathrm{Si}(\mathrm{Li})$ detectors arranged in 10 evenly spaced planes [11-14]. Each detector has a cylindrical shape of $\sim 10 \mathrm{~cm}$ diameter and $\sim 2.5 \mathrm{~mm}$ thickness and it is segmented into eight strips of equal area. The required operational temperature of $\sim-40^{\circ}$ is achieved with an oscillating heat pipe system $[15,16]$. The readout is performed with a dedicated ASIC [17]. In each plane $2 \times 2$ detectors are grouped into a module and 36 modules are arranged in a $6 \times 6$ array. The support structure of the planes is made of aluminum. A schematic view of the instrument is shown in Figure 1. 


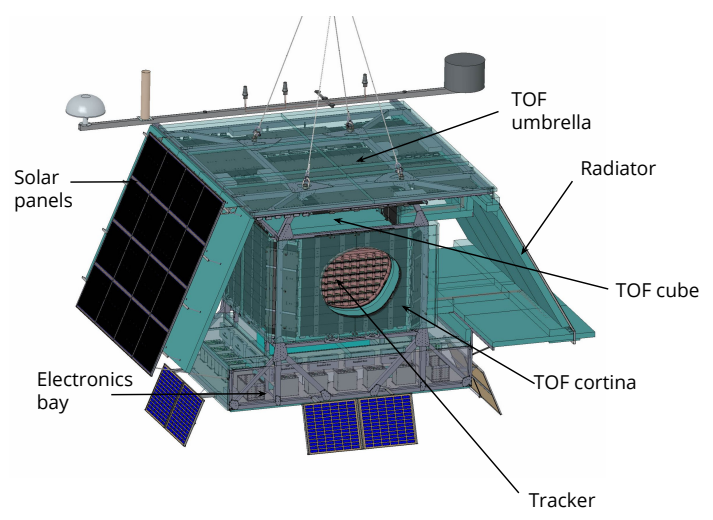

Figure 1: Schematic view of the GAPS experimental apparatus.

The detection principle is based on the obsevation of the annihilation products of the incoming antinucleus (hereafter called "primary particle" or just "primary"). The primary, after being slowed down by its ionization losses, can substitute an atomic electron (mostly in a silicon detector or in the aluminum frame) and form an exotic atom. The exotic atom then decays through a series of atomic transitions emitting characteristic X-rays [8], and the antinucleus finally annihilates with the target nucleus producing several secondary particles from a common vertex (mainly pions and protons). In order to discriminate antiproton nuclei from the cosmic ray background, a rejection power of at least $10^{6}$ is required, taking into account the relative particle abundances (e.g., [18]). To measure a possible antideuteron component an additional $10^{5}$ rejection factor is necessary to reject also antiproton background. A precise reconstruction of the event topology is required to achieve these discrimination performances.

\section{Reconstruction algorithm}

The reconstruction algorithm is designed to reconstruct the track of the primary particle, to identify the secondary particles produced in the annihilation, and to determine the position of the annihilation vertex.

The algorithm has been developed and validated using detailed Geant4 [19] simulations of the detector. Both the geometry and the response of the instruments have been implemented in the simulation. The position resolution of the ToF paddles (along the largest dimension) has been set to $4 \mathrm{~cm}$, consistent with the time resolution of better than $400 \mathrm{ps}$. The transfer function of the ASIC channels is also implemented to correctly reproduce the energy resolution of the silicon detectors ( $\sim 4 \mathrm{keV}$ below energy deposits of $\sim 100 \mathrm{keV}$, slowly increasing up to $100 \mathrm{keV}$ for deposits above 50 $\mathrm{MeV})$.

Before the actual reconstruction, a clustering procedure is performed on the hits in the ToF that are spatially and temporally consistent. A trigger condition is applied to select annihilation events: eight hits are required in the ToF, with at least three hits both in the outer and in the inner ToF. A cut on the deposited energy of the hits is also applied to reject both minimum ionizing particles and high- $Z$ particles. 


\subsection{Primary particle reconstruction}

The primary particle is identified assuming that it is the first particle hitting the outer and inner ToF. The first hit in the outer ToF and the first one in the inner ToF are used to build the initial track estimation, then a track-following approach is used: the initial track is extrapolated to the next detector plane, then the hits in that plane are associated to the primary if they satisfy the following requirements:

1. Spatial consistency: the intersection of the track with the plane $\left(\mathbf{p}_{i}\right)$ is calculated with its estimated uncertainty $\delta_{i}$. The estimated multiple scattering displacement is also included in the error. A hit in that plane is included if its distance from $\mathbf{p}_{i}$ is less than $\alpha_{i}(\beta) \cdot \delta_{i}$, where $\alpha_{i}(\beta)$ is a $\beta$-dependent coefficient.

2. Energy consistency: the spatially consistent hit with the highest $\mathrm{d} E / \mathrm{d} x$ (with $\mathrm{d} x=\rho \cdot \mathrm{d} l$, where $\mathrm{d} E$ is the energy deposit, $\rho$ is the density of the material and $\mathrm{d} l$ is the length of the track intersection in the active hit volume) are added to the primary track if $E_{i}>E_{i-1} / v_{i}(\beta)$, where $E_{i}$ is the energy deposit of the hit, $E_{i-1}$ is the deposited energy in the previous plane (or in the inner ToF in case of the first tracker plane), and $v_{i}(\beta)$ is a $\beta$-dependent coefficient.

3. Upper limit to distance between hits: a hit that is both spatially and energetically consistent but is more distant than $\gamma_{i}(\beta)$ from the previously added hit is discarded to avoid adding hits that are beyond the annihilation vertex.

Every time a hit from a plane is added, the primary track is estimated again with a least-squares minimization on all the associated hits. This method is then iterated through all the tracker planes. The coefficients $\alpha_{i}(\beta), v_{i}(\beta)$ and $\gamma_{i}(\beta)$ are chosen for each plane to ensure a $\sim 98 \%$ selection efficiency of the primary hits according to Monte Carlo simulations.

\subsection{Secondary tracks finding algorithm}

The remaining hits that are not associated with the primary are used to search for secondary tracks. Two algorithm were developed: a global approach based on the Hough-3D transform algorithm [20] and a custom algorithm specifically developed for this experiment. Since the latter was found to have better efficiency and vertex resolution (as will be shown in Section 4), it was chosen as the main reconstruction algorithm, and it is the one that will be described in this section.

A scan with a step of length $2 \mathrm{~cm}$ is performed along the primary track, starting from its entrance in the tracker volume until the projected exit: from every scan point $\mathbf{p}, 1281$ trajectories, isotropically distributed over the solid angle, are traced and the hits that are intercepted by a track are associated to it. Then, the track with the largest number of associated hits is selected and the search is repeated with the remaining hits. This method is iterated until there are no more tracks intercepting at least two hits. For each $\mathbf{p}$ the quantity

$$
Q(\mathbf{p})=\prod_{k=1}^{N(\mathbf{p})} \frac{n_{k}}{n}
$$

is evaluated, where $N(\mathbf{p})$ is the number of tracks found from $\mathbf{p}, n_{k}$ is the number of hits associated to the $k^{\text {th }}$ track and $n$ is the total number of hits. The value $\mathbf{p}_{\text {min }}$ that minimizes $\log Q(\mathbf{p})$ is chosen, and its track candidates are fitted with a least-squared method on their associated hits. 


\subsection{Annihilation vertex determination}

The annihilation vertex can be reconstructed if at least the primary track and one secondary track were found. The vertex position $\mathbf{p}_{\text {vert }}$ is found by minimizing the quantity

$$
\chi^{2}=\sum_{k=1}^{N}\left[\frac{d_{k}(\mathbf{p})}{\delta_{k}}\right]^{2}
$$

where $N$ is the number of tracks, $d_{k}(\mathbf{p})$ is the distance of the $k^{\text {th }}$ track from $\mathbf{p}$ and $\delta_{k}$ is the associated error evaluated from the covariance matrix of the fitted track parameters. Since spurious hits can still be wrongly associated to the tracks, the procedure is iterated again after doing some corrections:

1. The tracks with a distance from the vertex of greater than $20 \mathrm{~cm}$ are rejected, and the annihilation vertex is fitted again with the remaining tracks. This cut selects $98 \%$ of the tracks originating from the annihilation vertex according to Monte Carlo simulations.

2. The search for secondary tracks is done again around the previously estimated vertex, and a new vertex position is found.

3. If the primary track has associated hits beyond the vertex, they are removed from it and the fit of the track and the vertex is repeated.

4. If the annihilation vertex is found in a $\mathrm{Si}(\mathrm{Li})$ strip, the corresponding hit is associated to all the tracks and the fit of all the tracks and the vertex is repeated.

The stopping time of the primary particle can be estimated by back-propagating the secondary tracks that have at least two time measurements. The primary particle is excluded from this calculation: the extrapolation of the stopping time using the $\beta$ measured in the ToF does not take into account the slowdown of the primary due to the energy losses in the inner ToF and in the tracker, so a shorter time would be obtained with respect to the real one. Secondary particles are mostly lighter and relativistic, so their time extrapolation is more precise with respect to the primary. A reconstructed annihilation event of an antideuteron with $\beta=0.28$ is shown in Figure 2, where the comparison between the simulated and the reconstructed tracks can be appreciated.

\section{Reconstruction performance}

The performance of the primary reconstruction was estimated from the capability to associate the right hits and from the resolution of the measured velocity $(\beta)$. The difference between the true and the reconstructed number of primary hits is shown in Figure 3 for antiproton, antideuteron and antihelium-3 primaries. Most of the events $(\sim 85 \%)$ have no more than one wrongly associated hit. The $\beta$ resolution is $\sim 5 \%$ for particles entering from the top umbrella, while it is $\sim 20 \%$ for particles from the side walls. The big difference between the resolution for particles from the umbrella and from the sides is due to the shorter distance between the lateral inner and outer ToF $(\sim 35 \mathrm{~cm})$ with respect to the top ones $(\sim 90 \mathrm{~cm})$. However, most of the Galactic cosmic rays will enter from the top, while the majority of the particles entering from the sides are of atmospheric origin. 

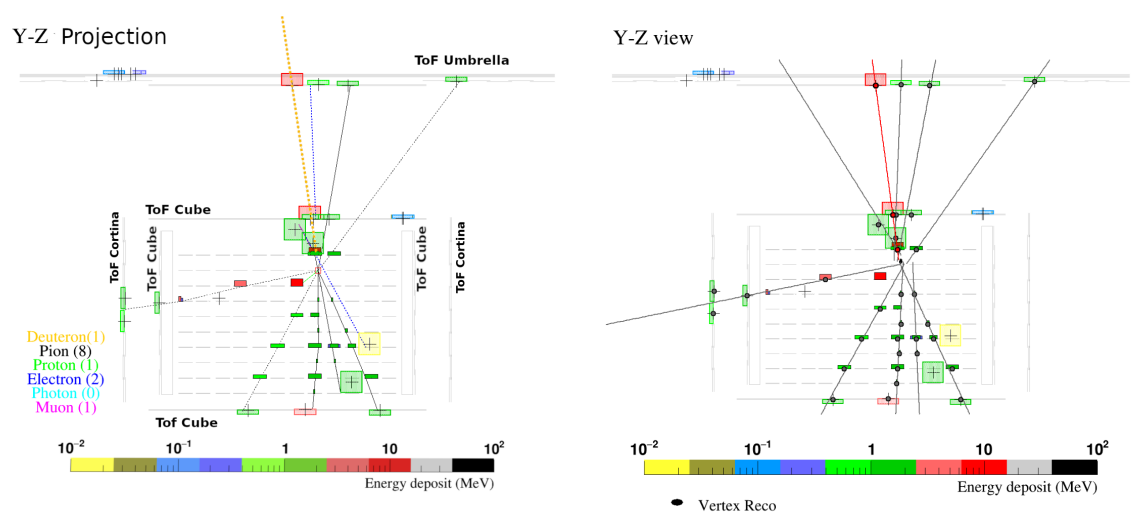

Figure 2: Schematic view of an antideuteron annihilation event (side view). Gray boxes represent the active parts of the detector. On the left side, the simulated tracks are drawn, with a different color for each particle species (solid lines for particles, dashed lines for antiparticles). Each colored box represents a hit, where the color indicates the amount of the energy deposition. On the right side, the reconstructed tracks and the associated hits are shown as gray lines/points (red for the primary) The small black ellipse represents the $95 \%$ confidence interval of the fitted annihilation vertex.

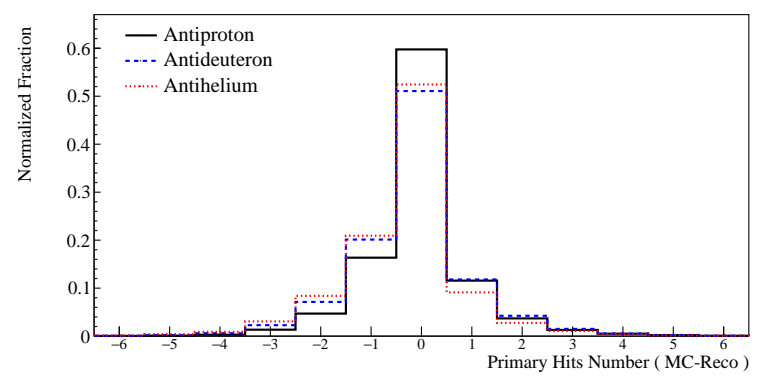

Figure 3: Difference between the number of primary hits according to Monte Carlo information and to reconstruction for antiprotons (solid line), antideuterons (dashed line) and antihelium-3 (dotted line).

The vertex reconstruction efficiency is estimated by selecting a sample of simulated antinuclei annihilating in the tracker volume according to simulation. The efficiency is then calculated requiring the existence of a reconstructed vertex. Figure 4 shows the reconstruction efficiency for different antinuclei as a function of the generated $\beta$. A value of about $90 \%$ is found for all particles and velocities. The efficiency of the Hough $3 \mathrm{D}$ algorithm is roughly $15 \%$ worse and it is also shown for comparison. The vertex position resolution is defined as the absolute distance between the true and the reconstructed vertex. The distribution of the distance is shown in Figure 5 for antiprotons, antideuterons, and antihelium-3. The distributions have a peak around $1 \mathrm{~cm}$, with $68 \%$ of the events within 9-12 cm The resolution of the Hough3D algorithm appears to be worse and it is shown for reference. Good spatial resolution is fundamental for the reconstruction of the trajectory and path length of the primary particle, which are needed for the discrimination of the various antinucleus species. As a reference, antideuterons incident vertically with $\beta<0.4$ annihilate more than $12 \mathrm{~cm}$ 


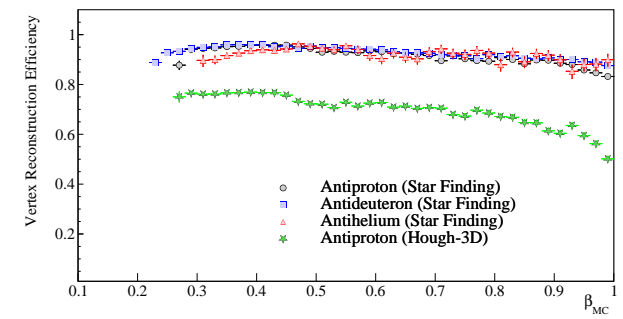

Figure 4: Vertex reconstruction efficiency as a function of the generated $\beta$ for antiprotons (circles), antideuterons (squares) and antihelium-3 (triangles). The efficiency of the Hough3D algorithm is also shown for comparison (stars)

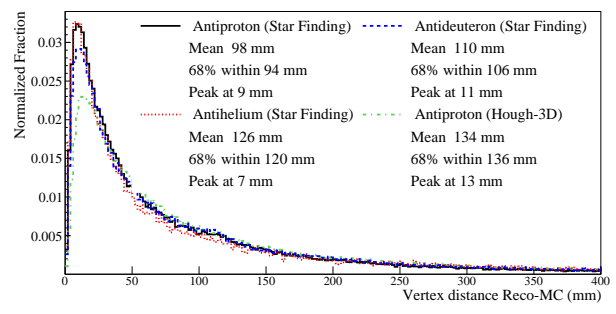

Figure 5: Distance between the vertex from reconstruction and from Monte Carlo information for antiprotons (solid line), antideuterons (dashed line) and antihelium-3 (dotted line). The performance of the Hough3D algorithm is also shown for comparison (dashed-dotted).

deeper in the tracker with respect to an antiproton with comparable velocity. The stopping time resolution exhibits an RMS of about $10 \%$.

\section{Acknowledgments}

This work is supported in the U.S. by NASA APRA grants (NNX17AB44G, NNX17AB45G, NNX17AB46G, and NNX17AB47G) and in Japan by JAXA/ISAS Small Science Program FY2017. P. von Doetinchem received support from the National Science Foundation under award PHY1551980. H. Fuke is supported by JSPS KAKENHI grants ( JP17H01136 and JP19H05198 ) and Mitsubishi Foundation Research Grant 2019-10038. K. Perez and M. Xiao are supported by HeisingSimons award 2018-0766. F. Rogers is supported through the National Science Foundation Graduate Research Fellowship under Grant No. 1122374. Y. Shimizu receives support from JSPS KAKENHI grant JP20K04002 and Sumitomo Foundation Grant No. 180322. This work is supported in Italy by Istituto Nazionale di Fisica Nucleare (INFN) and by the Italian Space Agency through the ASI INFN agreement no. 2018-28-HH.0: "Partecipazione italiana al GAPS - General AntiParticle Spectrometer". The technical support and advanced computing resources from the University of Hawaii Information Technology Services - Cyberinfrastructure are gratefully acknowledged.

\section{References}

[1] A. Cuoco, M. Krämer and M. Korsmeier, Novel Dark Matter Constraints from Antiprotons in Light of AMS-02, Phys. Rev. Lett. 118 (2017) 191102 [1610.03071].

[2] M.-Y. Cui, Q. Yuan, Y.-L.S. Tsai and Y.-Z. Fan, Possible dark matter annihilation signal in the AMS-02 antiproton data, Phys. Rev. Lett. 118 (2017) 191101 [1610.03840].

[3] F. Donato, N. Fornengo and P. Salati, Antideuterons as a signature of supersymmetric dark matter, Phys. Rev. D 62 (2000) 043003.

[4] N. Fornengo, L. Maccione and A. Vittino, Dark matter searches with cosmic antideuterons: status and perspectives, JCAP 09 (2013) 031 [1306.4171]. 
[5] H. Fuke and et al., Search for cosmic-ray antideuterons, Phys. Rev. Lett. 95 (2005) 081101.

[6] K. Mori, C.J. Hailey, E.A. Baltz, W.W. Craig, M. Kamionkowski, W.T. Serber et al., A Novel antimatter detector based on x-ray deexcitation of exotic atoms, Astrophys. J. 566 (2002) 604 [astro-ph/0109463].

[7] C.J. Hailey, An indirect search for dark matter using antideuterons: the GAPS experiment, New J. Phys. 11 (2009) 105022.

[8] GAPS collaboration, Antideuteron Sensitivity for the GAPS Experiment, Astropart. Phys. 74 (2016) 6 [1506. 02513].

[9] GAPS collaboration, Cosmic antihelium-3 nuclei sensitivity of the GAPS experiment, Astropart. Phys. 130 (2021) 102580 [2012.05834].

[10] S. Quinn, Recent Progress on the GAPS Time of Flight System, 36th International Cosmic Ray Conference (ICRC 2019), USA, Wisconsin, Madison (2019) .

[11] K. Perez et al., Fabrication of low-cost, large-area prototype Si(Li) detectors for the GAPS experiment, Nucl. Instrum. Meth. A 905 (2018) 12 [1807.07912].

[12] M. Kozai, H. Fuke, M. Yamada, K. Perez, T. Erjavec, C. Hailey et al., Developing a mass-production model of large-area si(li) detectors with high operating temperatures, Nuclear Instruments and Methods in Physics Research Section A: Accelerators, Spectrometers, Detectors and Associated Equipment 947 (2019) 162695.

[13] F. Rogers and et al., Large-area si(li) detectors for $x$-ray spectrometry and particle tracking in the GAPS experiment, Journal of Instrumentation 14 (2019) P10009.

[14] N. Saffold et al., Passivation of Si(Li) detectors operated above cryogenic temperatures for space-based applications, Nucl. Instrum. Meth. A 997 (2021) 165015 [2102.06168].

[15] H. Fuke, S. Okazaki, H. Ogawa and Y. Miyazaki, Balloon Flight Demonstration of an Oscillating Heat Pipe, J. Astron. Inst. 06 (2017) 1740006.

[16] S. Okazaki et al., Meter-scale multi-loop capillary heat pipe, Applied Thermal Engineering 141 (2018) 20.

[17] V. Scotti, A. Boiano, L. Fabris, M. Manghisoni, G. Osteria, E. Riceputi et al., Front-end electronics for the GAPS tracker, PoS ICRC2019 (2020) 136 [1909.01682].

[18] M. Boezio, R. Munini and P. Picozza, Cosmic ray detection in space, Prog. Part. Nucl. Phys. $112(2020) 103765$.

[19] GEANT4 collaboration, GEANT4-a simulation toolkit, Nucl. Instrum. Meth. A 506 (2003) 250.

[20] P.V.C. Hough, Machine Analysis of Bubble Chamber Pictures, Conf. Proc. C 590914 (1959) 554. 


\section{Full Authors List: GAPS Collaboration}

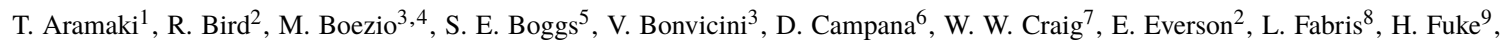
F. Gahbauer ${ }^{10}$, I. Garcia ${ }^{2}$, C. Gerrity ${ }^{11}$, C. J. Hailey ${ }^{10}$, T. Hayashi ${ }^{2}$, C. Kato ${ }^{12}$, A. Kawachi ${ }^{13}$, S. Kobayashi ${ }^{13}$, M. Kozai ${ }^{9}$, A. Lenni ${ }^{3,14}$, A. Lowell ${ }^{7}$, M. Manghisoni ${ }^{15,16}$, N. Marcelli ${ }^{17,18}$, B. Mochizuki ${ }^{7}$, S. A. I. Mognet $^{19}$, K. Munakata $^{12}$, R. Munini ${ }^{3,4}$, Y. Nakagami $^{20}$, J. Olson ${ }^{21}$, R. A. Ong ${ }^{2}$, G. Osteria ${ }^{6}$, K. Perez ${ }^{22}$, S. Quinn' ${ }^{2}$, V. Re ${ }^{15,16}$, E. Riceputi ${ }^{15,16}$, B. Roach ${ }^{22}$, F. Rogers ${ }^{22}$, J. A. Ryan ${ }^{2}$, N. Saffold ${ }^{10}$, V. Scotti ${ }^{6,23}$, Y. Shimizu ${ }^{24}$, M. Sonzogni ${ }^{15,16}$, R. Sparvoli ${ }^{17,18}$, A. Stoess $1^{11}$, A. Tiberio ${ }^{25}$, E. Vannuccini ${ }^{25}$, P. von Doetinchem $^{11}$, T. Wada ${ }^{20}$, M. Xiao 22 , M. Yamatami ${ }^{9}$, A. Yoshida ${ }^{20}$, T. Yoshida ${ }^{9}$, G. Zampa ${ }^{3}$, and J. Zweerink ${ }^{2}$

${ }^{1}$ Northeastern University, 360 Huntington Avenue, Boston, MA 02115, USA. ${ }^{2}$ University of California, Los Angeles, Los Angeles, CA 90095, USA. ${ }^{3}$ INFN, Sezione di Trieste, I-34149 Trieste, Italy. ${ }^{4}$ IFPU, I-34014 Trieste, Italy. ${ }^{5}$ University of California, San Diego, La Jolla, CA 90037, USA. ${ }^{6}$ INFN, Sezione di Napoli, I-80126 Naples, Italy. ${ }^{7}$ Space Sciences Laboratory, University of California, Berkeley, 7 Gauss Way, Berkeley, CA 94720, USA. ${ }^{8}$ Oak Ridge National Laboratory, Oak Ridge, TN 37831, USA. ${ }^{9}$ Institute of Space and Astronautical Science, Japan Aerospace Exploration Agency (ISAS/JAXA), Sagamihara, Kanagawa 252-5210, Japan. ${ }^{10}$ Columbia University, New York, NY 10027, USA. ${ }^{11}$ University of Hawaii at Manoa, Honolulu, HI 96822 USA. ${ }^{12}$ Shinshu University, Matsumoto, Nagano 390-8621, Japan. ${ }^{13}$ Tokai University, Hiratsuka, Kanagawa 259-1292, Japan. ${ }^{14}$ Universitá di Trieste, I-34127 Trieste, Italy. ${ }^{15}$ INFN, Sezione di Pavia, I-27100 Pavia, Italy. ${ }^{16}$ Universitá di Bergamo, I-24044 Dalmine (BG), Italy. ${ }^{17}$ INFN, Sezione di Rome "Tor Vergata", I-00133 Rome, Italy. ${ }^{18}$ Universitá di Roma "Tor Vergata", I-00133 Rome, Italy. ${ }^{19}$ Pennsylvania State University, University Park, PA 16802 USA. ${ }^{20}$ Aoyama Gakuin University, Sagamihara, Kanagawa 252-5258, Japan. ${ }^{21}$ Heliospace Corporation, Berkeley, CA 94710, USA. ${ }^{22}$ Massachusetts Institute of Technology, Cambridge, MA 02139, USA. ${ }^{23}$ Universitá di Napoli "Federico II", I-80138 Naples, Italy. ${ }^{24}$ Kanagawa University, Yokohama, Kanagawa 221-8686, Japan. ${ }^{25}$ INFN, Sezione di Firenze, I-50019 Sesto Fiorentino, Florence, Italy.

Web version: gaps1.astro.ucla.edu/gaps/authors/ 